\title{
Stellar and Ionized Gas Kinematics of Peculiar Virgo Cluster Galaxies
}

\author{
Juan R. Cortés ${ }^{1}$, Jeffrey D. P. Kenney ${ }^{2}$ and Eduardo Hardy ${ }^{3}$ \\ 1 Departamento de Astronomía, Universidad de Chile jcortes@das.uchile.cl \\ 2 Astronomy Department, Yale University kenney@astro.yale.edu \\ 3 National Radio Astronomy Observatory ehardy@nrao.edu
}

Summary. We present the results of the stellar and ionized gas kinematics of 13 bright peculiar Virgo cluster galaxies. The stellar velocity field are mostly consistent with a rotation pattern, but some of them shows interesting features such as; Sshaped stellar isovelocity contours in NGC 4064, and signatures of kinematical distinct components in NGC 4429, and NGC 4698. This latter galaxy and NGC 4424 exhibit extremely low $(V / \sigma)^{*}$ values suggesting that these galaxies are the result of mergers. The ionized gas velocity fields are more disturbed than the stellar velocity fields, displaying non-circular motions. Most galaxies in the sample reveals kinematical signatures that can be associated to gravitational interactions such as; mergers or tidal interactions, being specially clear in the "truncated/compact" galaxies. Moreover, most of the sample galaxies show evidence for both gravitational interactions, and ICM-ISM stripping. Thus the evolution of a significant fraction of cluster galaxies is likely strongly impacted by both effects.

\section{Introduction: Morphological evolution of Cluster galaxies}

It is well known that the environment affects the morphological types of galaxies in clusters. Observational facts such as the "morphology-environment" relation, "Butcher-Oemler effect", as well as the results of the MORPHS collaboration (Dressler et al. 1997), suggest that galaxies in clusters evolve morphologically, with spirals becoming lenticular and redder as the result of environmental effects.

Several mechanisms have been proposed for driving galaxy evolution, including processes that affect the gas and not the existing stellar stellar content (e.g., ICM-ISM stripping; Gunn \& Gott 1972; Schulz \& Struck 2001; Vollmer et al. 2001), and those interactions (Toomre \& Toomre 1972) that affect both the star and the gas (e.g., mergers; Hernquist 1992) However, it is still largely unknown which processes do actually occur and which among them are dominant in driving the morphological evolution of cluster galaxies.

Detailed studies of the stellar and ionized gas kinematics could help to discriminate between interacting processes. With this purpose in mind, we make a study of the stellar and ionized gas kinematics of thirteen peculiar Virgo cluster galaxies using integral-field spectroscopy techniques. Peculiar galaxies are natural targets for studying galaxy evolution, since they are "snapshots" 
of the galaxy evolution process. Moreover, the Virgo cluster is an ideal place for making this kind of studies, since it has a moderately dense ICM, is dynamically young with on-going sub-cluster mergers and infalling galaxies, and it has a significant population of galaxies characterized by truncated star formation morphologies (Koopmann \& Kenney 2004).

\section{The galaxy sample}

The sample consists in thirteen peculiar Virgo cluster galaxies spanning a variety of optical morphologies. Morphological selection was made using the $\mathrm{R}$, and $\mathrm{H} \alpha$ atlas of Virgo cluster galaxies of Koopmann et al. (2001), whereas the kinematical selection made use of the published $\mathrm{H} \alpha$ kinematics on 89 Virgo cluster spirals by Rubin et al. (1999). While the sample selection is not uniform, it is designed to include bright Virgo spirals whose peculiarities are most poorly understood, and to include representatives of the different $\mathrm{H} \alpha$ types identified by Koopmann \& Kenney (2004).

\section{Morphology of Peculiar Virgo Cluster galaxies}

Optical imaging in the $\mathrm{B}$, and $\mathrm{R}$-band and $\mathrm{H} \alpha$ narrow band was obtained using the Kitt Peak WIYN $3.5 \mathrm{~m}$ telescope with the Mini-mosaic imager. A summary of the observed morphologies is displayed in figure 1. The results show that six galaxies of the sample display disturbed outer stellar disks (NGC 4293, NGC 4351, NGC 4424, NGC 4569, NGC 4606 and NGC 4651 ), suggesting the action of gravitational interactions. Signatures of triaxial structures such as bars and lenses are found in NGC 4064, NGC 4450, and NGC 4457, whereas gross deviations from ellipticity such as heart-shaped isophotes, and non-elliptical isophotes are found in NGC 4293, NGC 4424, NGC 4429, NGC 4606, and NGC 4694.

At least, six galaxies have disturbed dust distributions; NGC 4064, NGC 4293, NGC 4424, NGC 4569, NGC 4606, and NGC 4694. Most of them show disturbed outer stellar disks, so they are probably the result of gravitational interactions. On the other hand, three galaxies do not exhibit any optical evidence for a recent gravitational interaction. These are NGC 4429, NGC 4457, and NGC 4580.

Most of the galaxies exhibit a depletion in their ionized gas, presenting truncated $\mathrm{H} \alpha$ distributions. NGC 4457, and NGC 4569 have truncated disks and peculiar $\mathrm{H} \alpha$ arms, suggesting an ongoing or recent ICM-ISM interaction. NGC 4580 has a truncated $\mathrm{H} \alpha$ disk with no peculiar $\mathrm{H} \alpha$ arm or $\mathrm{H} \alpha$ asymmetry, but it does have strong stellar spiral arms in the outer disk suggesting a recent ICM-ISM interaction. In the rest of the sample the cause of the depletion is not well understood with the present data, although most of them could have suffered the action of ICM wind sometime during their 
lifetime, as most of the galaxies in clusters, excepting the case of NGC 4651, which displays a fairly normal $\mathrm{H} \alpha$ disk.

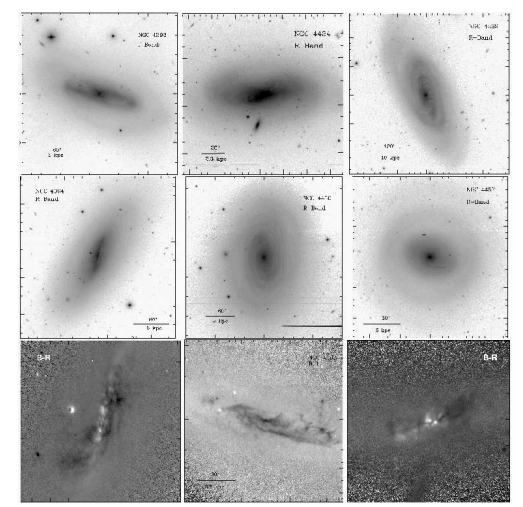

Fig. 1. Morphology of peculiar Virgo cluster galaxies. Top row: galaxies with disturbed outer stellar isophotes ;NGC 4293, NGC 4424 and NGC 4569. Middle row: barred and lensed galaxies; NGC 4064, NGC 4450, and NGC 4457. Bottom row: disturbed dust distribution in NGC 4064, NGC 4293 and NGC 4424.

\section{Stellar and Ionized gas kinematics}

We have mapped the stellar velocity fields, stellar velocity dispersion fields, and ionized gas velocity fields using the DensePak Integral-field unit installed at the $3.5 \mathrm{~m}$ WIYN telescope at Kitt Peak. Each galaxy was observed in the 4500 to $5000 \AA$, allowing us to obtain the ionized gas kinematics using the $\mathrm{H} \beta$ and $[\mathrm{O}$ III $] \lambda \lambda 4959,5007$ lines, and the stellar kinematics using the Magnesium triplet.

The stellar velocity fields in the galaxy sample are mostly consistent with a rotational pattern, but they also exhibit a variety of interesting structures (Figure 2). Some galaxies display misalignments between the photometric and kinematical major axes, which suggest the presence of non-axisymmetric structures, starting from the clear kinematical misalignments in the stellar kinematics of NGC 4293 to the S-type shape found in the isovelocity contours of NGC 4064 as consequence of the existence of the stellar bar, which contrasts with the $\mathrm{CO}$ velocity field (Cortés et al. 2006; Figure 2) which displays strong non-circular motions consistent with the infalling of gas to the center.

Signatures of a cold stellar disk (Figure 2) are found in NGC 4429, they characterize by peaks in velocity field correlated with peaks in $h_{3}$, and coincident with an undisturbed and axisymmetric dust disk, sugggesting that this cold stellar disk was probably formed by gas accretion. Finally a remarkable 
twisting in the isovelocity contours is found in NGC 4698 (Figure 2) which corresponds to a second kinematical component identified previously as an orthogonally rotating core by Bertola et al. (1999).

The ionized gas velocity fields, also exhibit a variety of structures, and in general look more disturbed than the stellar velocity fields. Non-circular motions are found in galaxies such as NGC 4064, NGC 4351, and NGC 4457. In this last galaxy, their are specially important along the anomalous $\mathrm{H} \alpha$ arm (Figure 2), suggesting the action of ICM-ISM stripping on a tilted gas disk (e.g; Schulz \& Struck 2001). Signatures of counter-rotation in the gas are found in NGC 4424, and evidence of a warped gas disk is found in NGC 4698 (Figure 2).
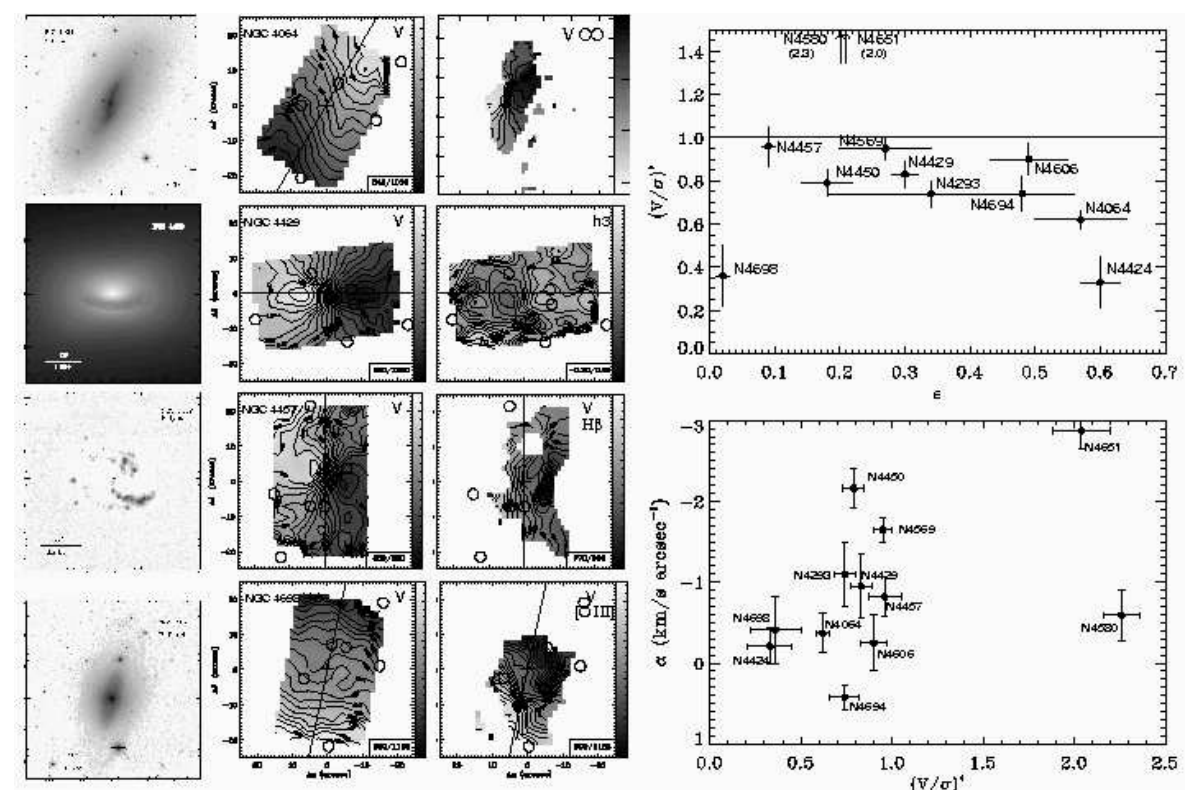

Fig. 2. Stellar and Ionized gas kinematics. Left : Stellar and ionized gas velocity fields for a set of sample galaxies. Right: $(V / \sigma)^{*}-\epsilon$ diagram, and $(V / \sigma)^{*}$ versus the slope of the velocity dispersion $\alpha$ for all the sample galaxies.

To understand the kinematics of the sample galaxies within the inner 2 kpc, we constructed a $(V / \sigma)^{*}-\epsilon$ diagram which is shown in Fig. 2. This shows that NGC 4580, and NGC 4651 are systematically well above the oblate isotropic rotator line, showing that their inner kinematics are dominated by the disk. On the other hand, NGC 4424, and NGC 4698 are extraordinary cases, lying well below the line and indicating that these objects have more elliptical-like kinematics favoring a merger scenario. We also have found that $(V / \sigma)^{*}$ correlates with the slope of the slope of the stellar velocity dispersion $\alpha$ 
(Figure 2), suggesting that dynamically hot systems tend to have flat velocity dispersion profiles in their inner $3 \mathrm{kpc}$. The fact that some of these have small $C_{30}$, all the Truncated/Compact galaxies defined by Koopmann \& Kenney (2004), and some with large $C_{30}$ suggest that the origin of this correlation should be due to an external cause, in particular to mergers.

\section{Morphological evolution in Peculiar cluster galaxies}

Our results in the morphology and kinematics indicate that most of the sample galaxies show evidence for both ICM-ISM stripping and gravitational interactions. Two sample galaxies show evidence for recent ICM-ISM stripping, but no strong recent gravitational interaction, just one galaxy show evidence for a recent minor merger but no ICM-ISM stripping, and one does not show any evidence of recent gravitational interaction or ICM-ISM stripping.

With the present evidence, results suggest that gravitational interactions play an important role in altering the morphologies toward a more lenticular stage (e.g. Bournaud et al. 2004) and driving gas inwards, but also drive gas outwards in the form of tidal tails (e.g. Barnes \& Hernquist 1991), so they are not responsible for the gas depletion in outer parts. ICM-ISM stripping seems to play a key role in the depletion of gas in the outer disk. Moreover, gravitational interaction can facilitate the action of ICM wind over the ISM (e.g. NGC 4424). ICM-ISM stripping plays an important role in "pre-process" spiral galaxies in the core of the cluster, where merger or gravitational interactions are more unlikely due to the high relative velocities. Finally, these combined effects seems to be crucial in objects with "Truncated/Compact" star formation as NGC 4064, and NGC 4424.

\section{References}

1. J. E. Barnes, \& L. Hernquist: ApJ, 370, L65 (1991)

2. F. Bournaud, F. Combes, \& C. J. Jog: A\&A, 418, L27 (2004)

3. F. Bertola, E. M. Corsini, J. C. Vega-Beltrán et al: ApJ, 519, L127 (1999)

4. J. R. Cortés, J. D. P. Kenney, \& E. Hardy: AJ, 131, 747 (2006)

5. A. Dressler, A. Jr. Oemler, W. J. Couch et al: ApJ, 490, 577 (1997)

6. J. E. Gunn, \& J. R. Gott: ApJ, 176, 1 (1972)

7. L. Hernquist: ApJ, 400, 460 (1992)

8. R. A. Koopmann, J. D. P. Kenney, \& J. Young: ApJS, 135, 125 (2001)

9. R. A. Koopmann, \& J. D. P. Kenney: ApJ, 613, 866 (2004)

10. V. C. Rubin, A. H. Waterman, \& J. D. P. Kenney: AJ, 118, 236 (1999)

11. S. Schulz, \& C. Struck: MNRAS, 328, 185 (2001)

12. A. Toomre, \& J. Toomre: ApJ, 178, 623 (1972)

13. B. Vollmer, V. Cayatte, C. Balkowski et al: ApJ, 561, 708 (2001) 